\title{
Design and analysis of a novel permanent magnet synchronous machine with axial assisted magnets
}

\author{
NA WANG ${ }^{1,2, * \mathbb{D}}$, HONGWEI LAN ${ }^{1}$ and YINZHEN $\mathrm{LI}^{2}$ \\ ${ }^{1}$ School of Mechatronic Engineering, Lanzhou Jiaotong University, Lanzhou, People's Republic of China \\ ${ }^{2}$ School of Traffic and Transportation, Lanzhou Jiaotong University, Lanzhou, People's Republic of China \\ e-mail: wangna@mail.lzjtu.cn
}

MS received 7 April 2018; revised 3 June 2019; accepted 9 August 2019

\begin{abstract}
The paper proposes a novel permanent magnet synchronous machine (NPMSM) with one radial permanent magnet synchronous machine (RPMSM) in the center and two axial permanent magnet synchronous machines (APMSMs) on both sides. The two APMSMs take advantage of the stator end winding spaces of the RPMSM and provide additional output power without increasing the whole machine volume. The emphasis of this study is on the unique rotor structure design and its merits for producing additional output power. The 3D finite element method (FEM), coupling with the equivalent circuit method is used in the study for designing the NPMSM. And the NPMSM prototype test results match well with the calculated results.
\end{abstract}

Keywords. Novel permanent magnet synchronous machine; radial permanent magnet synchronous machine; axial permanent magnet synchronous machine; finite element method; equivalent circuit method.

\section{Introduction}

As the heart of electric vehicles (EVs), the electric machines have attracted more and more attention. Different electric machine topologies for EVs are analyzed and compared, and the results show that the PMSMs have the best performance in the efficiency and power density. Many scholars and research institutes have made researches on PMSMs [1, 2].

There are various rotor topologies available for PMSMs, and the rotor topologies and parameters can lead to different performances of the EV electric machines [3, 4]. Recently, extensive studies have been conducted on the magnetic flux concentration [5-8]. Therefore, it is possible to increase the PMSMs power density with proper rotor structure.

In this paper, a novel permanent magnet synchronous machine (NPMSM) is proposed. The proposed NPMSM features a radial permanent magnet synchronous machine (RPMSM) in the center and two axial permanent magnet synchronous machines (APMSMs) on both sides. The two APMSMs take advantage of the stator end winding spaces of the RPMSM and provide additional output power without increasing the whole machine volume. The RPMSM and APMSMs share the same rotor core and the permanent magnets on the rotor are tangentially and axially magnetized, respectively. The radial air gap flux density can be increased by the axially magnetized permanent magnets.

*For correspondence

Published online: 05 September 2019
The axially magnetized permanent magnets not only provide additional radial fluxes for the RPMSM, but also provide the axial fluxes for both APMSMs, therefore the output power of the NPMSM is increased.

\section{Operating principle of NPMSM}

The structure of the NPMSM proposed is shown in figure 1. The NPMSM has three separated stators, one is the radial stator in the middle, and the other two are axial stators located on both sides of the radial stator. The rotor is surrounded by the three stators, and rotates around an axis. The tangentially magnetized permanent magnets (TMPMs) are inserted in the rotor core. The axially magnetized permanent magnets (AMPMs) are attached on both end surfaces of the rotor core. The radial air gap is formed by the rotor core outer cylindrical surface and the radial stator inner cylindrical surface; and both axial air gaps are formed between the rotor core end surfaces and the axial stators surfaces. The axial stators are fixed in the front and rear cover respectively. And the axial stators have their own winding.

Figure 2 is a perspective view showing a rotor and the permanent magnets arrangement.

The NPMSM rotor comprises the rotor core, TMSMs and AMPMs. The TMPMs are magnetized in the circumferential direction of the axis, and the AMPMs are magnetized in the axial direction of the axis. 


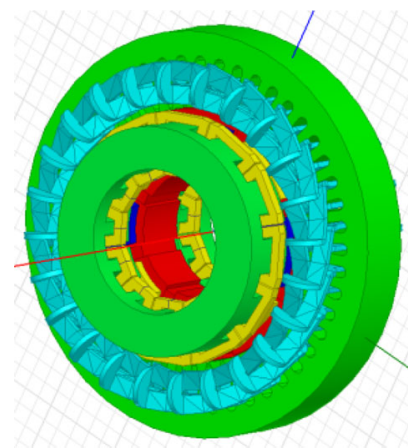

Figure 1. The NPMSM structure.

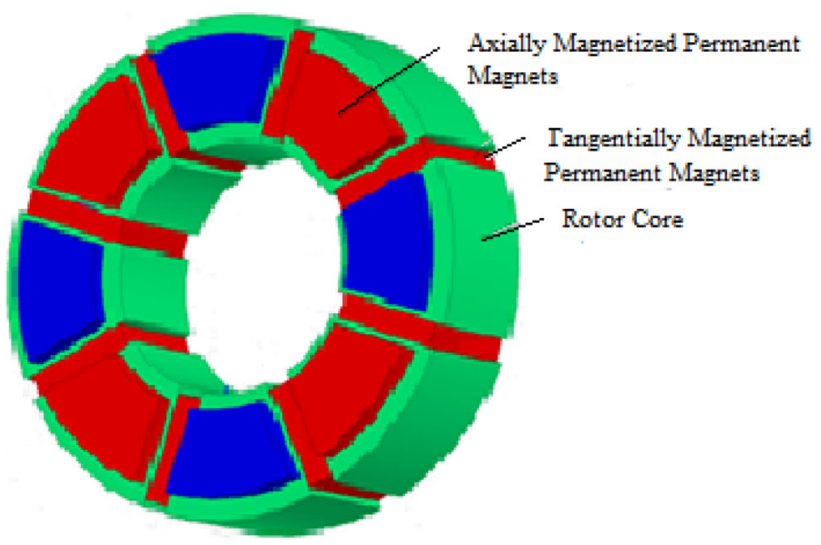

Figure 2. The NPMSM rotor structure.

Figure 2 shows that the TMPMs which are circumferentially magnetized are inserted in the rotor core, and the AMPMs which are axially magnetized are disposed on both the end sides of the rotor core. The pole direction of the permanent magnets are in the same polarity (same $\mathrm{N}$ poles or same $\mathrm{S}$ poles).

As shown in figure 3 , the TMPMs and the AMPMs are arranged staggeredly on a periphery. And AMPMs are arranged in pairs axially. Each two TMPMs which are circumferentially adjacent have the same polarity. And the AMPMs between two TMPMs have the same polarity.

The rotor structure makes full use of the spaces under thestator winding of RPMSM, and improves the NPMSM output power within the same volume capacity.

\section{Magnetic equivalent circuit analysis of the NPMSM}

Using the magnetic equivalent circuit analysis is the most common method for electric machine design.

In order to better understand the operating principles, an ideal magnetic circuit analysis is helpful and necessary. The magnetic equivalent circuit for the tangentially permanent magnet synchronous machine (TPMSM) is constructed with the assumption that the permeability of the iron is infinity.

Figure 4 shows the simple structure of TPMSM for one pole.

Figure 5 shows the magnetic equivalent circuit of TPMSM.

The air gap reluctance of the one pole $R_{\delta}$

$$
R_{\delta}=\frac{k_{c} \delta}{\frac{\mu_{0} \pi R_{r} L_{r}}{p}}
$$

where $R_{\delta}$ is the average magnetic reluctance, $\delta$ is the air gap length, $\mu_{0}$ is the permeability of air $\left(4 \pi \times 10^{-7}\right)$, and $k_{c}$ is the Carter coefficient $[9,10]$.

$$
k_{c}=\left[1-\frac{w_{s}}{\tau_{s}}+\frac{4 \delta}{\pi \tau_{s}} \ln \left(1+\frac{\pi w_{s}}{4 \delta}\right)\right]^{-1}
$$

where $w_{s}$ is the slot width, and $\tau_{s}$ is the stator pitch, in practical situations, the Carter's coefficient $k_{c}$ should be greater than 1 considering the increase of the air gap magnetic reluctance. As $\frac{\delta}{\tau_{s}}$ increases and $\frac{w_{s}}{\tau_{s}}$ decreases, the Carter coefficient converges to 1 .

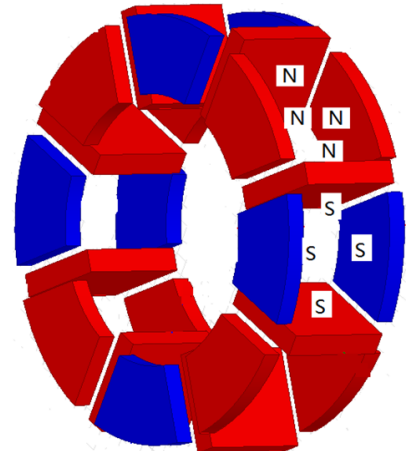

Figure 3. The structure of the TMPMs and AMPMs arrangement.

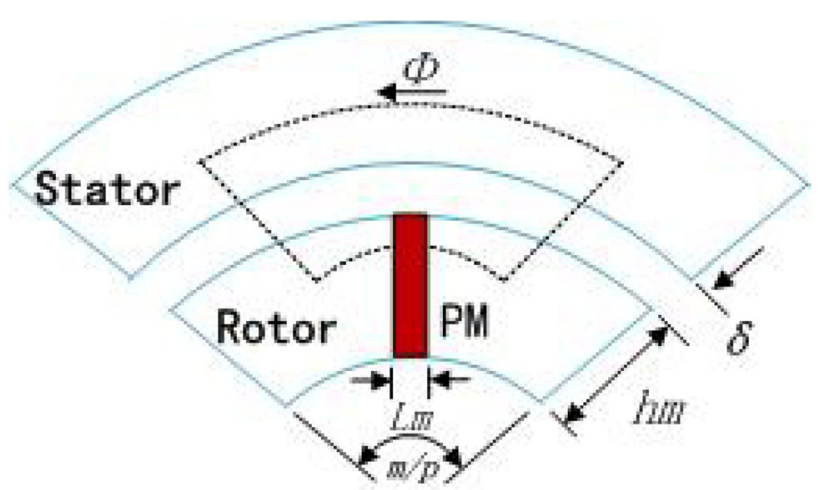

Figure 4. One pole structure of TPMSM. 


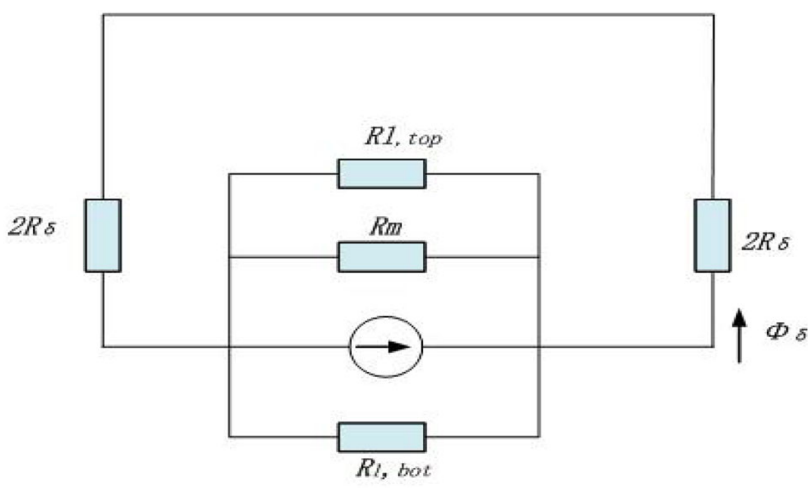

Figure 5. The Equivalent Circuit of TPMSM.

The remanence flux from the permanent magnet is

$$
\begin{gathered}
\emptyset_{r}=B_{r} L_{m} L_{r} \\
R_{p m}=\frac{h_{m}}{\mu_{0} \mu_{r} L_{m} L_{r}}
\end{gathered}
$$

where the $h_{m}$ is the PM thickness, and $L_{m}$ is the PM width, $\mu_{r}$ is the relative permeability of the permanent magnet. $L_{r}$ is the rotor core axial length, and the PM axial length is considered the same with it. The bridges and ribs of the rotor ensure the necessary mechanical performance to keep the deformation of the rotor from centrifugal force. The leakage at the bridges or the ribs of the rotor is not negligible. Generally speaking, the bridges and the ribs are fully saturated under operating situation, and under this condition the bridges behave like air [9, 10], so the reluctance at the top and bottom bridges of the rotor in figure 4 are:

$$
\begin{aligned}
& R_{l, t o p}=\frac{\frac{\pi}{2} h_{m}}{\mu_{0} t_{b, t o p} L_{r}} \\
& R_{l, b o t}=\frac{\frac{\pi}{2} h_{m}}{\mu_{0} t_{b, b o t} L_{r}}
\end{aligned}
$$

where $\frac{\pi}{2} h_{m}$ is the approximate distance for traveling leakage flux $[9,10]$, and $t_{b, t o p}$ and $t_{b, b o t}$ are the thickness of the to and bottom part of the bridges, respectively.

Assuming that the bridges are saturated at a constant flux density $B_{\text {sat }}$, then each leakage flux through the top and bottom bridges are:

$$
\begin{aligned}
& \emptyset_{l, t o p}=B_{s a t} t_{b, t o p} L_{r} \\
& \emptyset_{l, b o t}=B_{s a t} t_{b, b o t} L_{r}
\end{aligned}
$$

With the simple circuit analysis of figure 5, the air gap flux is

$$
\frac{1}{R_{\sigma}}=\frac{1}{R_{m}}+\frac{1}{R_{l, t o p}}+\frac{1}{R_{l, b o t}}
$$

$$
\emptyset_{\delta}=\left(\emptyset_{r}-\emptyset_{l, t o p}-\emptyset_{l, b o t}\right) \frac{R_{\sigma}}{R_{\sigma}+4 R_{\delta}}
$$

Therefore, the air gap flux density can be calculated simply by dividing the air gap flux by the air gap surface.

$$
B_{\delta}=\frac{\emptyset_{\delta}}{\frac{\pi R_{r} L_{r}}{2 P}}
$$

where $R_{r}$ is the stator inner radius. $P$ is the pole pairs. The magnetic circuit model of the TPMSM is constructed in figure 6.

Figure 7 shows the magnetic equivalent circuit of NPMSM, where the $R_{m \_ \text {tang }}$ is the reluctance, and the $\emptyset_{t}$ is the remanence flux of the TMPMs. $R_{r 1}, R_{r 2}$ are the rotor core reluctances on both sides of the TMPMs. $R_{g_{\text {_rad } 1} \text {, }}$ $R_{g_{-} \text {rad } 2}$ are reluctances of the radial air gap between the rotor and rotor stator, and $R_{s \_r a d}$ is the reluctance of the radial stator core. The TMPMs magnetic fluxes travel from the N pole of TMPMs, and pass through the rotor core, the radial air gap, the radial stator core, then by another side radial air gap, the rotor core, to the adjacent $S$ pole of the TMPMs.

The $R_{a m 1}, R_{a m 2}, R_{a m 11}, R_{a m 22}$ are the AMPMs reluctances on the rotor both sides, and the $\emptyset_{a}$ is the remanence flux of the AMPMs. $R_{g \_a x i 1}, R_{g \_a x i 2}$ are the axial air gap reluctances on both sides of the rotor. $R_{s_{-} a x i}$ is the axial stator reluctance. The AMPMs magnetic fluxes travel from the $\mathrm{N}$ pole of the AMPMs, the axial air gap, the axial stator, to the adjacent air gap, to the adjacent $\mathrm{S}$ pole, the rotor core, the radial air gap, the radial stator, to adjacent radial air gap, the rotor core successively, finally to the AMPMs start point, forming the closed magnetic circuit.

The AMPMs provide magnetic effect that focuses on TPMSM, increase the radial air gap flux density, and then improve the power density of TPMSM.

In the NPMSM, additional AMPMs on both sides of the rotor provide more fluxes for the radial air gap, which travel from the radial air gap to the radial stator, returns from the adjacent poles of the rotor, then enters both the axial air gap to the axial stator on both sides to complete the magnetic circulation. The AMPMs not only add the radial air gap fluxes, but also form two axial permanent magnet

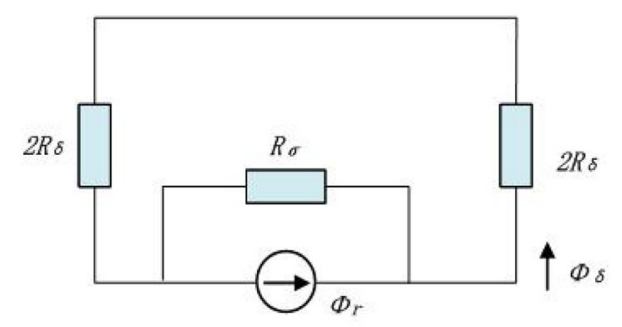

Figure 6. The simplified magnetic equivalent circuit of TPMSM. 


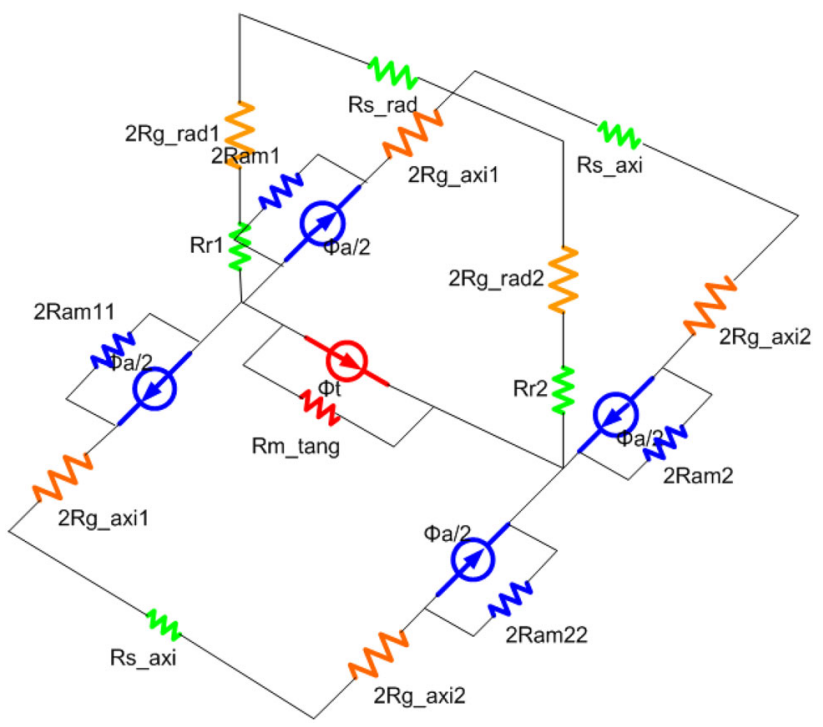

Figure 7. The magnetic equivalent circuit of NPMSM.

synchronous machines. These can improve the electric machine output power largely.

\section{Finite element method}

In a real electric machine, there is saturation of flux density in the stator and rotor. In order to calculate accurately, 3D FEM is used for the simulation and design.

Figure 8 is the simulation results with 3D FEM which showed the fluxes paths of the AMPMs and TMPMs, and

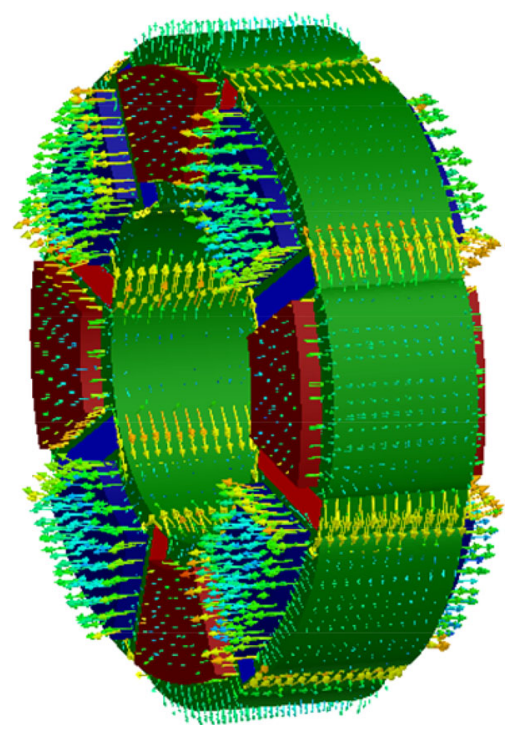

Figure 8. The flux paths of NPMSM.

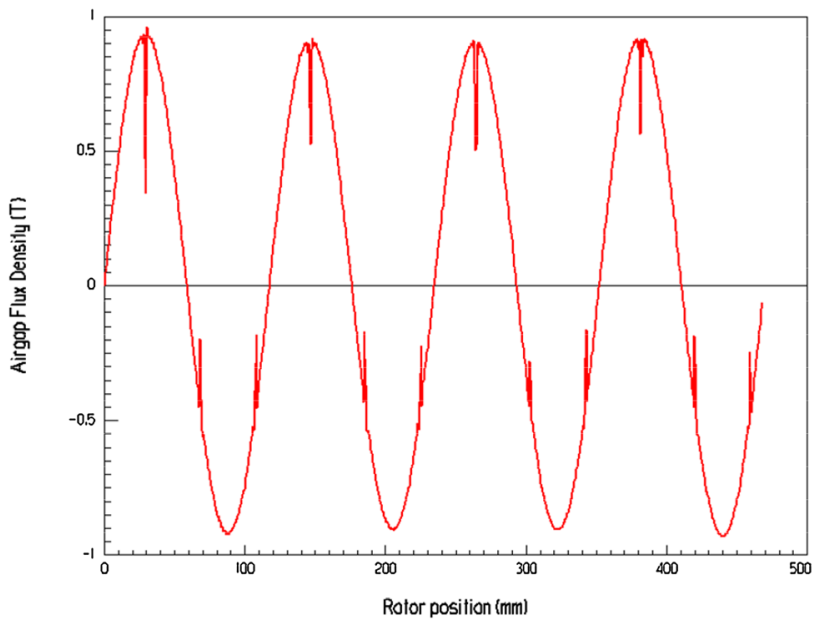

Figure 9. The simulation results of the radial air gap flux density.

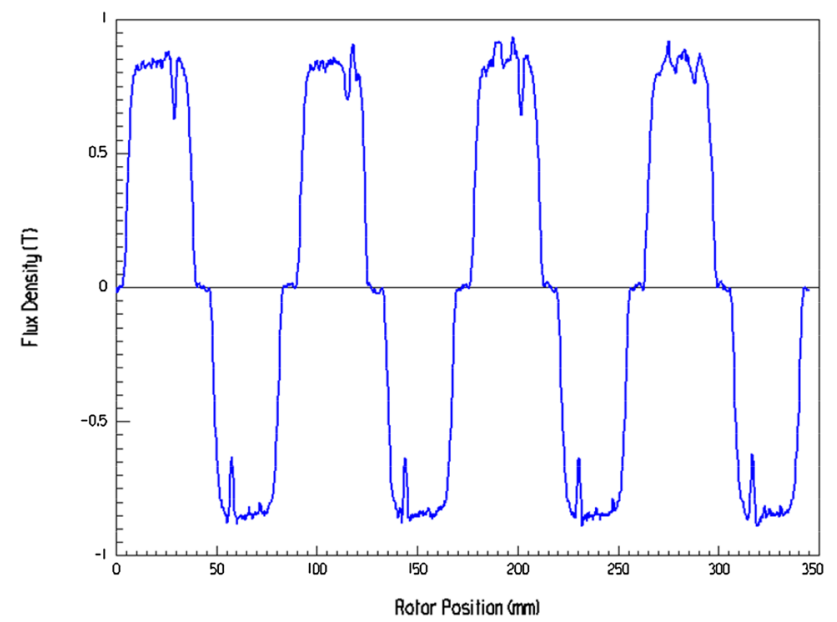

Figure 10. The simulation results of the axial air gap flux density.

we can see that the AMPMs boost the flux density of the radial air gap.

Figure 9 is the simulation results of the radial air gap flux density computed by 3D FEM when the TMPMs remanence $B_{r_{-} \text {rad }}=0.45 \mathrm{~T}$, AMPMs remanence $B_{r_{-} a x i}=1.2 \mathrm{~T}$.

The radial air gap flux density obtains relative high value with lower remanence $\mathrm{B}_{\mathrm{r}-\mathrm{rad}}$ of TMPMs, which reduces the cost of RPMSM. The AMPMs not only increase the radial air gap flux density, but also form two APMSMs. Figure 10 shows the simulation results of the axial air gap flux density with 3D FEM for the two APMSMs.

An actual NPMSM prototype is assembled.

Figure 11 depicts the machine assembly of the NPMSM prototype.

The back electromotive force (EMF) is a key parameter to evaluate the electric machine performance. Figure 12 presents the prototype testing platform and the measured A 


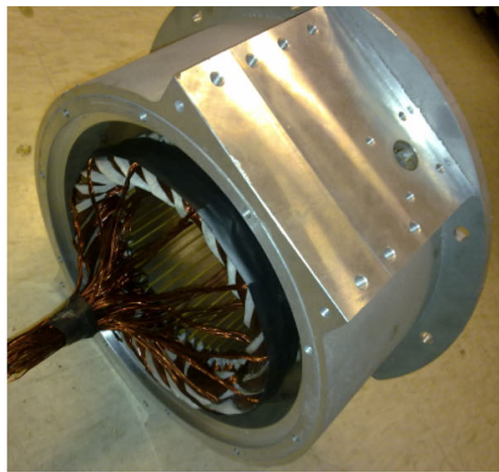

(a) RPMSM stator assembly

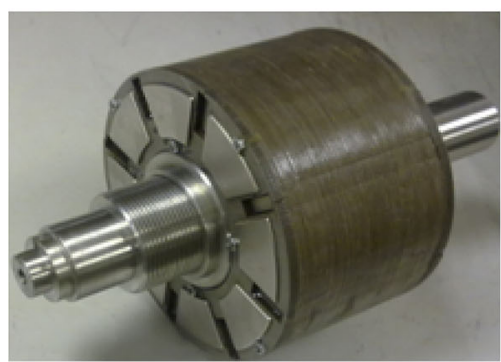

(b) The rotor assembly

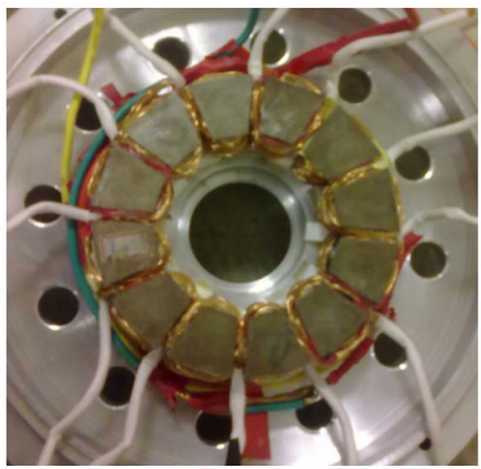

(c) APMSM stator assembly.

Figure 11. Photographs of the NPMSM prototype (a) RPMSM stator assembly, (b) the rotor assembly and (c) the APMSM stator assembly.

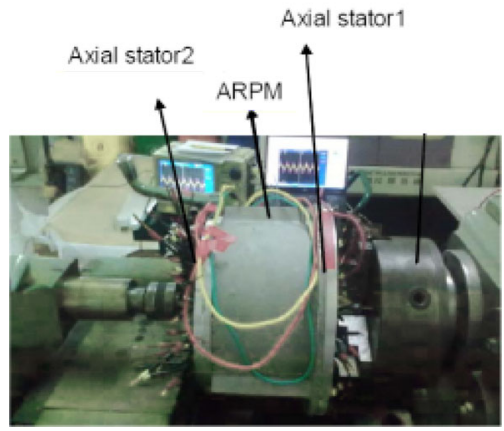

Figure 12. Experimental set for NPMSM.

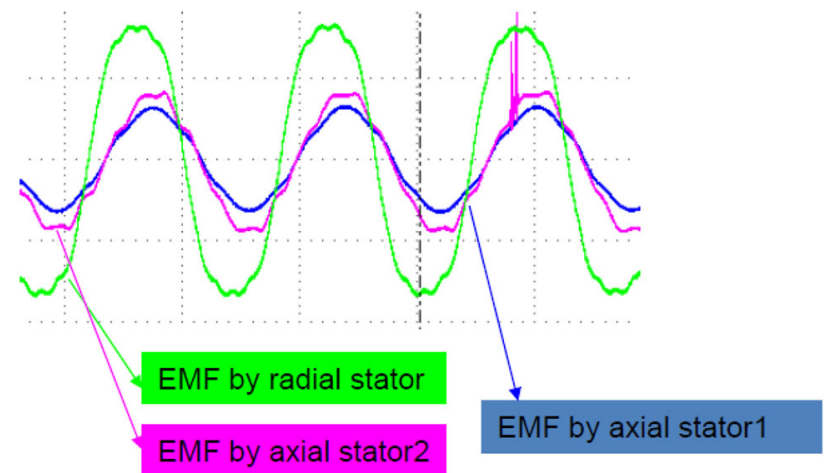

Figure 13. Experimental results of back EMF of NPMSM @ $900 \mathrm{rpm}$.

Table 1. The back EMF comparison of $3 \mathrm{D}$ simulation and test.

\begin{tabular}{lcc}
\hline Items & 3D FEM & Prototype test \\
\hline Speed (rpm) & 900 & 900 \\
$E_{\text {Axi1_peak }}$ & 3.69 & 2.76 \\
$E_{\text {Axi2_peak }}$ & 3.69 & 2.94 \\
$E_{\text {Rad_peak }}$ & 33.6 & 30.2 \\
$\frac{E_{\text {Axil_peak }}+E_{\text {Axi2_peak }}}{E_{\text {Rad_peak }}}$ & $22 \%$ & $19 \%$ \\
\hline
\end{tabular}

phase back EMF under $900 \mathrm{rpm}$ with no load is shown in figure 13.

Table 1 shows the comparison of the simulation results of 3D FEM and the test measured results. And the results show that the APMSMs on both sides of the rotor provide additional output power, which is about $22 \%$ of the TPMSM output power in the 3D FEM simulation and 19\% of the TPMSM output power in the test, which means that the NPMSM could increase the output power nearly $20 \%$ without increasing its volume. Clearly, the experimental tests have validated the 3D FEM simulation results.

\section{Conclusion}

In this study, we proposed the flux concentrated rotor structure which obtained the high radial air gap flux density with lower remanence $\mathrm{B}_{\mathrm{r}-\mathrm{rad}}$. In the application for electric vehicles, NPMSM was analyzed by equivalent magnetic circuit method and designed by 3D FEM. Both the equivalent magnetic circuit method and 3D FEM simulation method are very useful for the electric machine design. And the 3D FEM simulation results have been experimentally validated by the prototype. The simulation and test results showed that the AMPMs not only increase the radial air gap flux density, but also increase the output power with the APMSMs on both sides of the rotor. The NPMSM can obtain higher output power without increasing the machine volume. And the NPMSM proposed is suitable for the electric vehicles as the traction machine. 


\section{Appendix}

\section{Nomenclature}

$R_{\delta}$

$\delta$

$\mu_{0}$

$k_{c}$

$w_{s}$

$\tau_{s}$

$\emptyset_{r}$

$h_{m}$

$L_{m}$

$\mu_{r}$

$L_{r}$

$\frac{\pi}{2} h_{m}$

$t_{b, t o p}$

$B_{\text {sat }}$

$\emptyset_{l, t o p}$

$\emptyset_{l, b o t}$

$B_{\delta}$

$\emptyset_{\delta}$

$R_{r}$

$P$

$R_{m \_ \text {tang }}$

$\emptyset_{t}$

$R_{r 1}, R_{r 2}$

$R_{g \_r a d 1}, R_{g \_r a d 2}$

$R_{s \quad \text { rad }}$

$R_{a m 1}, R_{a m 2}, R_{a m 11}, R_{a m 22}$

$\emptyset_{a}$

$R_{g \_a x i 1}, R_{g \_a x i 2}$

$R_{S \_a x i}$

$B_{r}$

$R_{p m}$

$R_{l, t o p}$
The average magnetic reluctance

The air gap length

The permeability of air

$\left(4 \pi \times 10^{-7}\right)$

The Carter coefficient [9-10]

The slot width

The stator pitch

The remanence flux from the

permanent magnet

The PM thickness

The PM width

The relative permeability of the permanent magnet

The rotor core axial length

The approximate distance for

traveling leakage flux $[9,10]$

The thickness of the top part of the bridges respectively

A constant flux density

Each leakage flux through the top bridges

Each leakage flux through the bottom bridges

The air gap flux density

The air gap flux

The stator inner radius

The pole pairs

The reluctance

The remanence flux of the tangential permanent

magnets(TPM)

The rotor core reluctance on both sides of the TPM

The radial air gap reluctance on both sides of TPM

The reluctance of the radial

stator core

The axial permanent magnet

(APM) reluctance on the rotor both sides

The remanence flux of the APM

The axial air gap reluctance on

both sides of the rotor

The axial stator reluctance

The flux density of the

permanent magnet

The reluctance of the permanent magnet

The reluctance at the top bridge of the rotor
$R_{l, b o t}$

$\emptyset_{l, t o p}$

The reluctance at the bottom bridge of the rotor

Each leakage flux through the top bridge

$\emptyset_{l, b o t}$

$R_{m}$

Each leakage flux through the bottom bridge

The reluctance at the middle bridge of the rotor

Br_rad

$B_{r \_a x i}$ The flux density of the radial stator core

The flux density of the axial stator

\section{References}

[1] Kamiya M 2006 Development of traction drive motors for the Toyota hybrid system. IEEE Trans. IA. 126: 473-479

[2] Y Dai, Song L W and Cui S M 2007 Development of PMSM drives for Hybrid electric car applications. IEEE Trans. Magn. 43: 434-437

[3] Dorrell D G, Kniht A M, Popescu M, Evans L and Staton D A 2010 Comparison of different motor design drives for hybrid electric vehicles. In: IEEE Energy Conversion Congress and Exposition, Atlanta, GA, United States, pp. 3352-3359

[4] Goss J, Popescu M and Staton D 2013 A comparison of an interior permanent magnet and copper rotor induction motor in a hybrid electric vehicle application. In: International Electric Machines \& Drives Conference, Illinois, United States, pp. 220-225

[5] Kim H J, Kim D Y and Hong J P 2014 Structure of concentratedflux-type interior permanent-magnet synchronous motors using ferrite permanent magnets. IEEE Trans. Magn, 50: 1-4

[6] Zhao W, Lipo T A and Kwon B I 2014 Comparative study on novel dual stator radial flux and axial flux permanent magnet motors with ferrite magnets for traction application. IEEE Trans. Magn. 50: 1-4

[7] Rahman M M, Kim K T and Hur J 2014 Design and optimization of neodymium-free-spoke type motor with segmented wing-shaped PM. IEEE Trans. Magn. 50: 865-868

[8] Fukami T, Yasuhiro M, Takahito H, Shima K, Hanaoka R and Takata S 2008 Steady-state analysis of a permanentmagnet-assisted salient-pole synchronous generator. In: 18th International Conference on Electrical Machines, Wuhan, China, vol. 25, pp. 388-393

[9] Thelin P 1999 Calculation of the airgap flux density of PM synchronous motors with buried magnets including axial leakage and teeth saturation. In: 9th International Conference on Electrical Machines and Drives, Canterbury, UK, vol. 468, pp. 339-345

[10] Thelin P 2000 Analytical calculation of the airgap flux density of PM synchronous motors with buried magnets including axial leakage, tooth and yoke saturation. IEEE Power Electronics and Variable Speed Drives 475: 218-223 